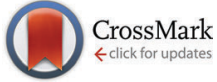

Cite this: Phys. Chem. Chem. Phys., 2016, 18, 3975

Received 28th August 2015,

Accepted 22nd December 2015

DOI: $10.1039 / c 5 c p 05143 f$

www.rsc.org/pccp

\section{A robust comparison of dynamical scenarios in a glass-forming liquid}

\author{
Alessandro Vispa, ${ }^{a}$ Sebastian Busch, ${ }^{b}$ Josep Lluis Tamarit, ${ }^{a}$ Tobias Unruh, ${ }^{c}$ \\ Felix Fernandez-Alonso de and Luis Carlos Pardo*a
}

\begin{abstract}
We use Bayesian inference methods to provide fresh insights into the sub-nanosecond dynamics of glycerol, a prototypical glass-forming liquid. To this end, quasielastic neutron scattering data as a function of temperature have been analyzed using a minimal set of underlying physical assumptions. On the basis of this analysis, we establish the unambiguous presence of three distinct dynamical processes in glycerol, namely, translational diffusion of the molecular centre of mass and two additional localized and temperature-independent modes. The neutron data also provide access to the characteristic length scales associated with these motions in a model-independent manner, from which we conclude that the faster (slower) localized motions probe longer (shorter) length scales. Careful Bayesian analysis of the entire scattering law favors a heterogeneous scenario for the microscopic dynamics of glycerol, where molecules undergo either the faster and longer or the slower and shorter localized motions.
\end{abstract}

\section{Introduction}

An understanding of the dynamics of glass-forming liquids at temperatures significantly above the glass transition still represents a much-coveted goal so as to establish a sound physical framework to explore the undercooled liquid state. Although we have experimental access to the equilibrium states of the liquid at high temperatures, the very nature of glasses makes it impossible to probe these at temperatures below the glass transition. Therefore, if any theory or explanation is to be extrapolated to understand the undercooled regime from knowledge of high-temperature equilibrium states, a consensus must first exist on the underlying physics of the liquid phase at temperatures above the glass transition. Such a consensus remains a challenge to the field, and often the analysis of experimental data is invariably colored by specific theoretical considerations. The primary aim of this work is to provide a detailed and critical appraisal of quasielastic neutron scattering (QENS) data by

\footnotetext{
${ }^{a}$ Grup de Caracterització de Materials, Departament de Física i Enginyeria Nuclear, ETSEIB, Universitat Politècnica de Catalunya, Diagonal 647, E-08028 Barcelona, Catalonia, Spain. E-mail: luis.carlos.pardo@upc.edu

${ }^{b}$ German Engineering Materials Science Centre (GEMS) at Heinz Maier-Leibnitz Zentrum (MLZ), Helmholtz-Zentrum Geesthacht GmbH, Lichtenbergstr. 1, 85747 Garching bei München, Germany

${ }^{c}$ Chair for Crystallography and Structural Physics, Friedrich-Alexander-Universität Erlangen-Nürnberg, Staudtstr. 3, 91058 Erlangen, Germany

${ }^{d}$ ISIS Facility, Rutherford Appleton Laboratory, Chilton, Didcot, Oxfordshire OX11 OQX, UK

${ }^{e}$ Department of Physics and Astronomy, University College London, Gower Street, London, WC1E 6BT, UK
}

invoking physical models which are as simple and general as possible. In doing so, we address two specific questions about the dynamics of liquid glycerol, a prototypical glass-forming liquid: the first one concerns how many distinct dynamical processes are present in glycerol in the sub-nanosecond (ns) regime. The second one concerns whether a HomoGeneous (dynamically driven, hereafter $\mathrm{HG})^{1,2}$ or HeTerogeneous (structurally driven, $\mathrm{HT})^{3-5}$ scenario better describes the QENS data. To avoid the common pitfalls associated with "classical" data-analysis procedures (e.g., use of additional parameters not justified by the information content of the data), we make extensive use of Bayesian inference methods.

Glycerol is one of the most-studied "classical" glass-forming liquids. ${ }^{6,7}$ The dynamics of glycerol have been studied by dielectric spectroscopy (DS), ${ }^{8-12}$ depolarized light scattering, ${ }^{13}$ nuclear magnetic resonance (NMR), ${ }^{14,15}$ and QENS. ${ }^{16-20}$ Moreover, molecular dynamics (MD) simulations have been performed in order to explore the dynamics of this glass-forming liquid. ${ }^{21} \mathrm{An}$ early and quite extensive analysis of QENS data on glycerol was performed by Wuttke et al. ${ }^{22-24}$ in the context of mode coupling theory (MCT). This study made extensive use of phenomenological Kohlrausch-Williams-Watts (KWW) line shapes to describe the slow dynamics. Very recently, Cicerone et al. ${ }^{4}$ have shown that the picosecond (ps) dynamics of glycerol and other glass-forming liquids might be studied by recourse to a twostate scenario where tightly and loosely caged domains coexist. Moreover, the liquid dynamics seem to be driven primarily by structural features. In what follows, we first examine the characteristic time scales of these processes. Since our experiments have been performed as a function of temperature, we will also 
compare relaxation times with those obtained from DS, in order to gain additional insights into the nature of $\beta_{\mathrm{JG}}$-relaxation phenomena. Finally, we ask whether our QENS data are better described by the HG or HT via recourse to two first-order models built with a minimum number of assumptions.

\section{Materials and methods}

\subsection{Measurements and data reduction}

All experiments were performed using a glycerol sample purchased from Sigma Aldrich with a purity greater than 99\%. QENS experiments were performed using the TOFTOF spectrometer at the Heinz Maier-Leibnitz Zentrum (MLZ) in Munich (Germany) using a resolution of $55 \mu \mathrm{eV}$ (full width at half maximum). ${ }^{25}$ The samples were kept in hollow aluminium cylinders with a sample layer thickness of $0.1 \mathrm{~mm}$. This thin layer ensures a transmission of $\sim 85 \%$, thereby rendering multiple scattering effects small. The sample temperature was controlled using a closed-cycle refrigerator, to collect QENS data at $T=292,316,345,380 \mathrm{~K}$, corresponding to $T_{\mathrm{g}} / T=0.65$, $0.60,0.55,0.50$.

The program FRIDA $^{26}$ was used for data reduction. From the time-of-flight of the neutrons, the energy transfer between the neutron and the sample was calculated; the measured double differential neutron scattering cross-section was transformed into the scattering function; an angle- and energy-dependent absorption correction was performed on both the sample measurements and the measurement of a vanadium standard which was used to determine the detector efficiencies and the instrumental resolution; the detector efficiencies were normalized; and finally the momentum transfer $Q$ was calculated from the scattering angle and the energy transfer and slices of constant $Q$ were grouped together in bins of $0.1 \AA^{-1}$.

As shown in Fig. 1, the $Q$-range analyzed is between $0.6 \AA^{-1}$ and $1.3 \AA^{-1}$ where the coherent contribution is negligible. In energy, the spectra were evaluated in the whole accessible region.

\subsection{Data evaluation}

Following the seminal work of Sivia and Carlile, ${ }^{27,28}$ the analysis of the data has been performed using a Bayesian approach. Instead of simply finding the set of parameters that best fits the data and its related minimum $\chi^{2}$, all parameters $\left\{P_{i}\right\}$ and associated $\chi^{2}$ values compatible with the experimental data and associated uncertainties have been calculated using a Markov-chain Monte Carlo process. ${ }^{29,30}$ Using this method, model selection is then performed by considering the information content of entire $\chi^{2}$ probability density functions (PDFs), as opposed to relying on a single value of the merit function or its reduced version. All data analysis presented herein has been performed using the FABADA and SIDRA ${ }^{46}$ software packages developed by our group. FABADA was used to fit parametric models to the data and has been thoroughly described before. ${ }^{29-31}$ SIDRA was used to fit QENS data with a free or parametrized distribution of modes so that the scattering function $S(Q, E)$

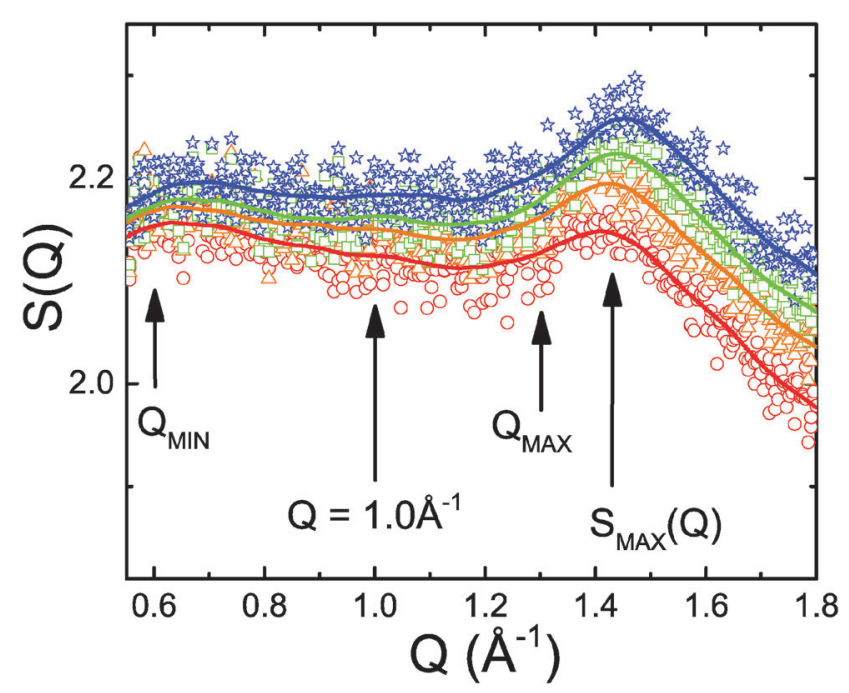

Fig. 1 Static structure factor $S(Q)$ for glycerol at $T=380 \mathrm{~K}$ (red circles), $345 \mathrm{~K}$ (orange triangles), $316 \mathrm{~K}$ (green squares) and $292 \mathrm{~K}$ (blue stars). The $Q$-range analyzed is $Q_{\min }=0.6 \AA^{-1}$ to $Q_{\max }=1.3 \AA^{-1}$. This region is sufficiently far away from the structure factor maximum $S_{\max }$ at around $1.4 \AA^{-1}$ to avoid strong contributions of coherent scattering. $Q=1.0 \AA^{-1}$ is shown because the dynamic range is maximal for this momentum transfer, and so is the amount of information present in the data. The solid lines are a guide to the eye.

can be described as a function of momentum ( $\hbar Q)$ and energy (E) transfer by: ${ }^{32}$

$$
S(Q, E)=A(Q) \delta(E)+\sum_{i=1}^{n} B_{i}\left(Q, \Gamma_{i}\right) \frac{1}{\pi}\left(\frac{\Gamma_{i}}{E^{2}+\Gamma_{i}^{2}}\right)
$$

where $\Gamma_{i}$ is the energy width of the $i$-th Lorentzian function and $B_{i}\left(Q, \Gamma_{i}\right)$ is the weight for each Lorentzian, being thus the collection of $\left\{B_{i}\left(Q, \Gamma_{i}\right)\right\}, i=1, \ldots, N$ the normalized probability distribution function describing the dynamics of the system. $B_{i}\left(Q, \Gamma_{i}\right)$ can be easily transformed to the time domain so that the intermediate scattering function $I(Q, t)$ can be written down as a distribution of simple exponential functions weighted by $B_{i}\left(Q, \tau_{i}\right){ }^{32}$ There are two main methods to obtain $B_{i}\left(Q, \tau_{i}\right)$, namely: from a model-free distribution that can be determined, for example, by a maximum entropy method; ${ }^{32}$ or from a parametrized line shape such as a Gaussian or the Fourier transform of a KWW distribution in the time domain. ${ }^{33}$ The maximum-entropy method has the drawback that a KWW distribution is equivalent to the sum of quasi-Gaussian modes, ${ }^{32}$ i.e., it is not possible to ascertain whether the data are described by one or more dynamical processes. For this reason, we have not adhered to this approach in the present work, restricting ourselves to parametrized Gaussian and KWW distributions.

In order to perform the analysis, the theoretical function $S(Q, E)$ must be convolved with the instrumental resolution $R(Q, E)$ in the energy dimension before comparison to the measured data. When the width of the theoretical function $\Gamma$ is close to the width of the energy bins of the data $\Delta E$, the convolution process artificially increases the broadening $\Gamma .^{34}$ This artifact is especially prominent when fitting a diffusion 
process, since in this case $\Gamma$ is proportional to $Q^{2}$. The calculated value of $\Gamma$ is therefore larger than the true one in the low $Q$ region. In other words, as soon as the line width $\Gamma$ is similar to the energy bin width $\Delta E$, a deviation from the $Q^{2}$ behaviour is observed which is not related to any physical process, but to a numerical problem of the convolution. In order to avoid this effect, the bin width must be chosen smaller than twice the extracted line width, i.e. $\Delta E \leq 2 \Gamma$. $^{34}$

\section{Results and discussion}

\subsection{Determination of the number of processes}

To answer our first question, i.e., how many dynamical processes are present in the measured dynamics of glycerol, a series of plausible (and widely used) models have been analyzed, namely: a two Lorentzian model (2L), a distribution of modes described by two Gaussian PDFs, and another one described by a KWW plus a Gaussian PDF ( $\mathrm{G}+\mathrm{KWW})$. We have also compared all of these two-component models with a simple three-Lorentzian model (3L) and, for the sake of consistency, also with a single KWW distribution. In all cases, fits to the data were performed after convolution with the spectral resolution of the instrument. Fig. 2 shows the results of this analysis and the resulting $\chi^{2}$ PDFs at $T=380 \mathrm{~K}$ and $Q=1.0 \AA^{-1}$. Model selection has been performed using the program FABADA $^{35-37}$ at $Q=1.0 \AA^{-1}$ because the dynamic range and, therefore, the information content of QENS spectra are maximal at this particular $Q$. This momentum transfer is dominated by incoherent scattering, as it lies significantly below the first and most pronounced maximum of the measured structure factor located at $Q_{\max }=$ $1.4 \AA^{-1}$ (see also Fig. 1).

Inspection of the resulting $\chi^{2}$ PDFs evinces that the $3 \mathrm{~L}$ model provides the best description of the experimental data (cf. Fig. 2). Although one might argue that this conclusion could very well be the result of an increase in the number of fit parameters relative to simpler models, inspection of the underlying and well-separated $\chi^{2}$ PDFs rules out this possibility in glycerol. In other words, the two-component model fits using any combination of parameters are invariably worse than those corresponding to the $3 \mathrm{~L}$ model.

Given the popularity of the G + KWW model, we have also performed a series of additional fits to obtain values for the 'stretching' parameter $\beta_{\mathrm{KWW}}$ at $Q=1.0 \AA^{-1}$ as a function of temperature. At the lowest (highest) temperature, we find $\beta_{\mathrm{KWW}} \approx 0.5(0.7)$. These values are in closer agreement with dielectric spectroscopy ${ }^{8-11}$ than previous QENS studies. ${ }^{23}$ Notwithstanding this encouraging result, we stress that our Bayesian analysis does not favour this particular model, as evidenced by the results shown in Fig. 2 .

\subsection{Model comparison: homogeneous vs. heterogeneous}

In order to gain further insights into the physical basis of the three processes described by the $3 \mathrm{~L}$ model in glycerol, we have performed further model selection on the entire scattering function $S(Q, E)$ accessible in the QENS experiments. The aim of this exercise is to elucidate whether, in addition to the expected translational diffusion of the molecular centre of mass, all molecules are subjected to: either two additional (and distinct) relaxation processes affecting the entire ensemble ( $\mathrm{HG})$; or if some molecules are moving faster than others giving rise to the so-called islands of mobility (HT). ${ }^{38,39}$ Mathematically the HG can be described by a multiplicative ansatz of dynamical processes in the time domain and, as such, it affects all molecules on the time scale of the $\alpha$-relaxation. The HT, on the other hand, is based on the arithmetic sum of two processes, each one affecting a certain percentage of molecules at $\alpha$-relaxation time scales. With these considerations in mind, the simplest (two-state) incoherent scattering functions for the HT and HG read, respectively,

$$
\begin{aligned}
S(Q, \omega)_{\mathrm{HG}}= & y_{0}+L_{\mathrm{D}} \otimes\left[a(Q) \cdot \delta(\omega)+(1-a(Q)) L_{\mathrm{a}}\right] \otimes[b(Q) \cdot \delta(\omega) \\
& \left.+(1-b(Q)) L_{\mathrm{b}}\right] \\
S(Q, \omega)_{\mathrm{HT}}= & y_{0}+L_{\mathrm{D}} \otimes\left\{P\left[a(Q) \cdot \delta(\omega)+(1-a(Q)) L_{\mathrm{a}}\right]\right. \\
& \left.+(1-P)\left[b(Q) \cdot \delta(\omega)+(1-b(Q)) L_{\mathrm{b}}\right]\right\} .
\end{aligned}
$$
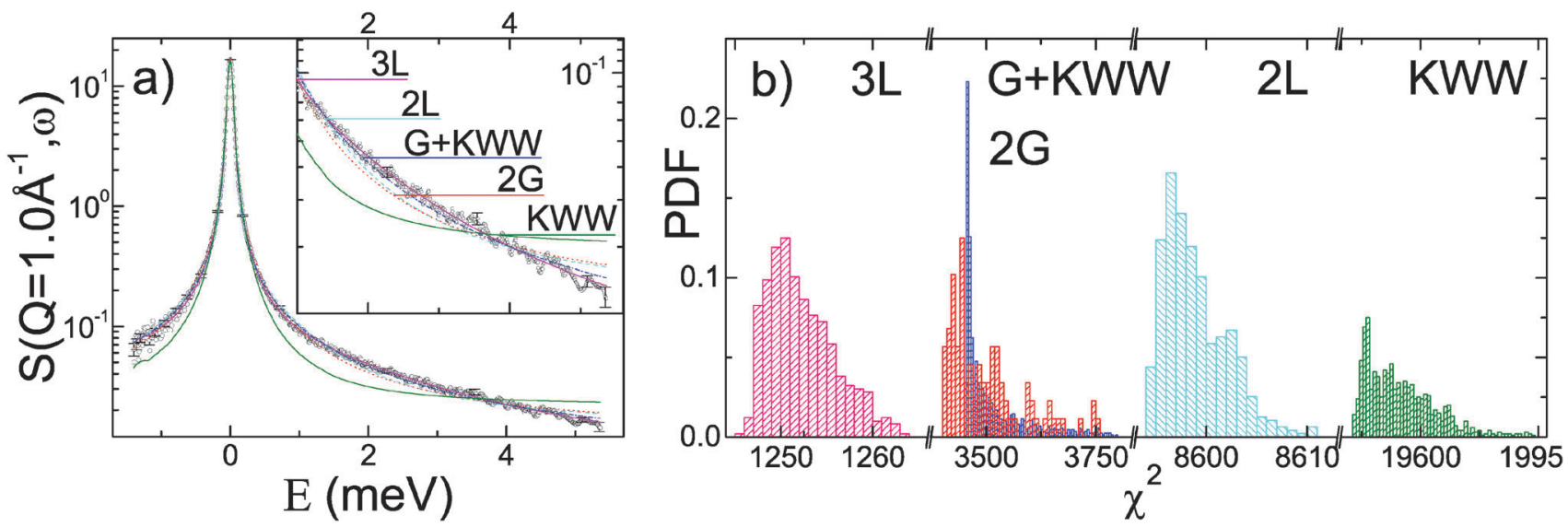

Fig. 2 (a) Data fits for glycerol at $T=380 \mathrm{~K}$ and $Q=1.0 \AA^{-1}$ using different models: two lorentzians (2L), three lorentzians (3L), a single KWW relaxation (KWW), KWW plus a Gaussian distribution of relaxation times (KWW $+\mathrm{G}$ ), and two Gaussian distributions (2G). Using the same colors, panel (b) shows the corresponding $\chi^{2}$ PDFs. 
where $L_{\mathrm{D}}$ is a Lorentzian line describing translational diffusion with a spectral width proportional to the square of the momentum transfer, that is, $\Gamma=D Q^{2}$, where $D$ is the translational diffusion coefficient. We note the intrinsic (linear) dependence of $D$ on $Q^{2}$, a relation that has been used to describe the experimental data as a function of $Q$ using a single fit parameter $D$. The remaining (localized) motions are described by $\left[P_{i}(Q) \cdot \delta(\omega)+\left(1-P_{i}(Q)\right) L_{P_{i}}\right]$ with $P_{i}=a, b$. The dependence of the form factors $a(Q)$ and $b(Q)$ on $Q$ provides a measure of the associated length scales for these motions.

In order to establish whether the spectral widths $\Gamma_{i}$ associated with localized motions are $Q$-independent, we have allowed these to vary across $Q$ at all temperatures. The results for $T=380 \mathrm{~K}$ are shown in the inset of Fig. 5 and demonstrate that, within experimental uncertainties, they are $Q$-independent. In view of the above, we have replaced a total of 16 parameters (2 Lorentzian widths across 8 independent $Q$-values) by $2 Q$-independent spectral widths which were then fit simultaneously across all $Q$ slices. This reduction in the number of adjustable parameters results in a drastic decrease in error bars. As explicitly shown by eqn (2) and (3), the use of $Q$-independent spectral widths to describe the localized modes does not imply that the resulting Lorentzian modes are of constant width, since convolution with the translational component necessarily leads to spectral features of width $\Gamma_{i}+D Q^{2}$.

The results of this analysis at two $Q$ values are shown in Fig. 3a (an overview over all fits are shown in Fig. 4), demonstrating that both scenarios can describe the QENS data well. The resulting $\chi^{2}$ PDFs are shown in Fig. $3 \mathrm{~b}$, where $\chi^{2}$ values correspond to the global values across all energy and momentum transfers probed by QENS. Fig. 3b serves to highlight a significant overlap of the $\chi^{2}$ PDFs for both models, which means that model selection in this particular case is certainly more ambiguous than in the previous case dealing with a determination of the total number of distinct dynamical processes. Although in this case the HT is preferred (both the minimum and maximum of the $\chi^{2}$ PDF are located at

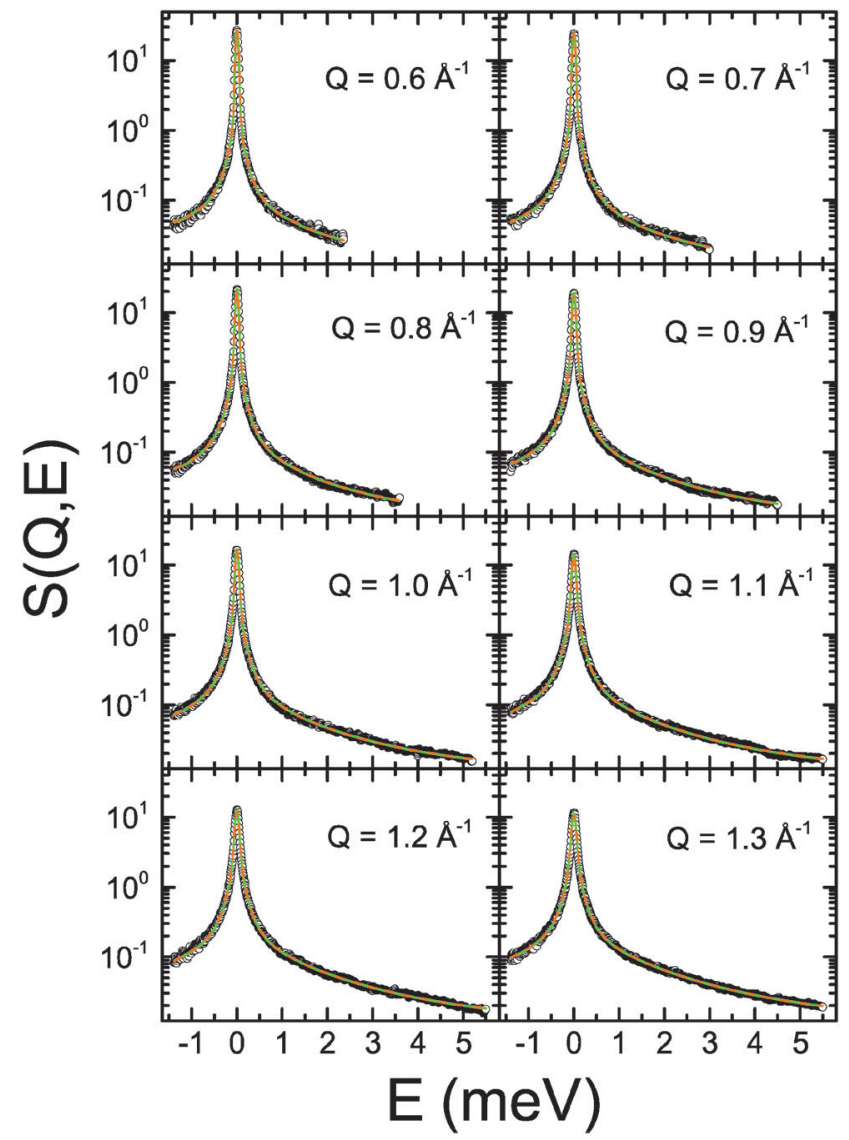

Fig. 4 The quasielastic scattering function $S(Q, E)$ of glycerol (at $380 \mathrm{~K}$ ) for all values of $Q$ evaluated in this work. Also shown are the best fits with the two models HG (solid green line) and HT (dashed orange line).

lower values), it is still possible that additional information encoded in an improved prior (or new measurements over a wider range of length and timescales) could change the above conclusion. In any case, given the information at hand, the HT is favored.
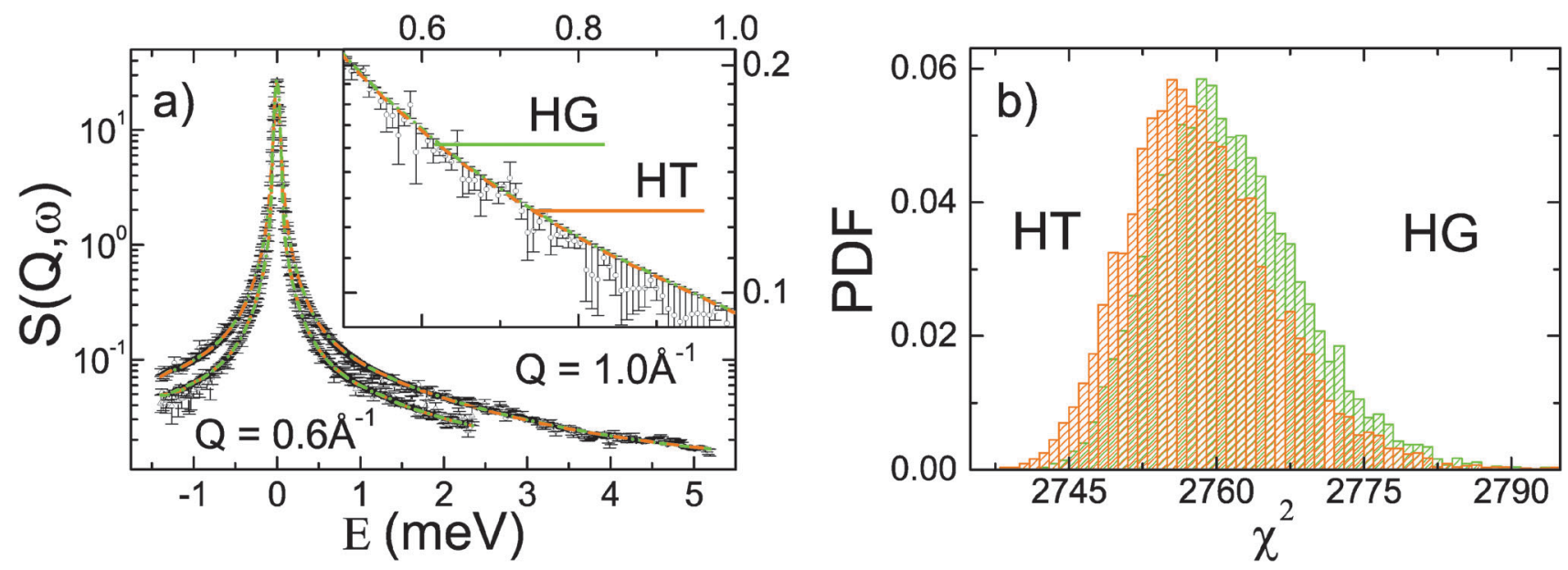

Fig. 3 (a) Representative QENS spectra of glycerol at $T=380 \mathrm{~K}$ and associated fits for the HT/HG (solid/dashed lines); (b) $\chi^{2}$ PDFs obtained from global fits over the entire scattering function as discussed in the main text. 


\subsection{Physical parameters extracted from the HG and HT models}

In both models, the peak is described by a Lorentzian line shape with a half width at half maximum (HWHM) $\Gamma_{\mathrm{d}}$ which is related to the diffusion coefficient $D$ by $\Gamma_{\mathrm{d}}=D Q^{2}$. The diffusion coefficient is obtained for each temperature and for the two tested models ( $c f$. Fig. 5). Across the whole temperature range, the value of the diffusion coefficient is basically independent of the employed model (HG or HT).

Fig. 6 reports the relaxation times $\tau_{i}=\hbar / \Gamma_{i}$ obtained for all three processes of the HT and HG model. First of all, we find that the obtained $\tau_{i} \mathrm{~S}$ values are independent of the model chosen and, therefore, their determination is robust. In addition to the relaxation times $\tau_{\text {slow }}$ and $\tau_{\text {fast }}$ associated with the two localized processes, we also show the relaxation time $\tau_{\max }$ at $Q_{\max }$, a dynamical quantity that describes the collective dynamics. As shown in Fig. 6, the temperature dependence of $\tau_{\max }$ is in good agreement with collective $\alpha$-relaxation data measured with DS. ${ }^{8-11}$ These two datasets deviate slightly from each other as the temperature is lowered because the timescales associated with translational diffusion increase and, as a result, they approach the intrinsic energy resolution of the QENS experiments. ${ }^{40}$

As discussed previously, ${ }^{41}$ the translational diffusion coefficients obtained from the QENS data are in line with literature values. Thus, we may conclude that our quantitative determination of translational diffusion coefficients is robust, further corroborating the physical validity of the priors defined by eqn (2) and (3). Concerning the localized modes, we find $\tau_{\text {fast }}=$ $0.2 \mathrm{ps}$ and $\tau_{\text {slow }}=5.5 \mathrm{ps}$, and both are insensitive to temperature.

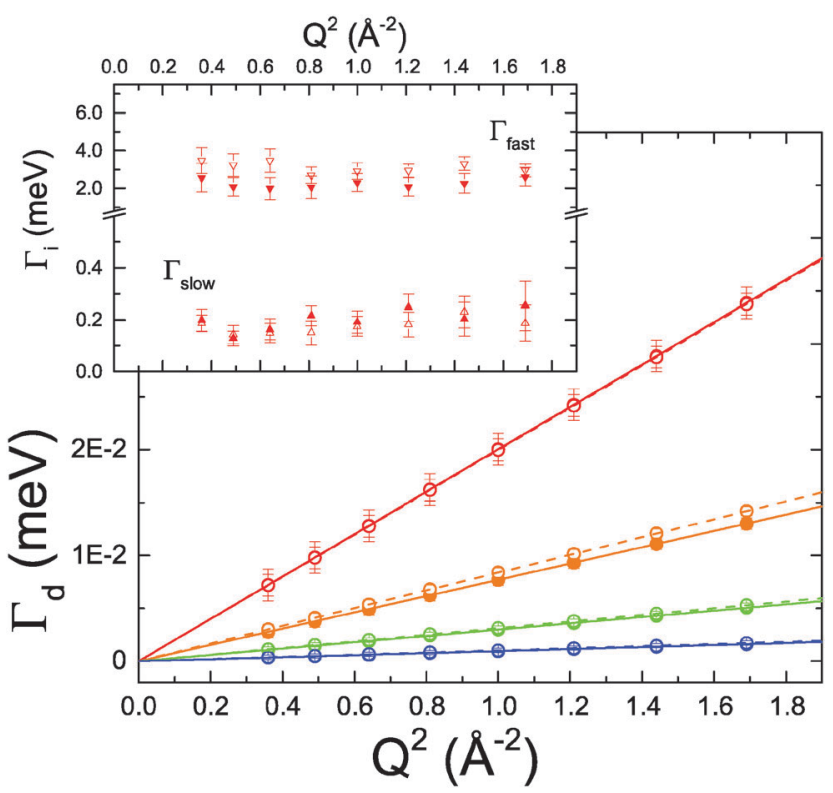

Fig. $5 Q$-dependent linewidth $\Gamma_{\mathrm{d}}$ (HWHM) of $S(Q, E)$ for different temperatures. At each temperature, a clear $Q^{2}$ dependence of $\Gamma_{\mathrm{d}}$ is observed, compatible with a diffusive motion. The lines show the best fits of the function $\Gamma_{\mathrm{d}}=D Q^{2}$ to the data. It is evident that diffusion coefficients $D$ obtained for the two models HG (full symbols and solid lines) and HT (open symbols and dashed lines) are very similar.

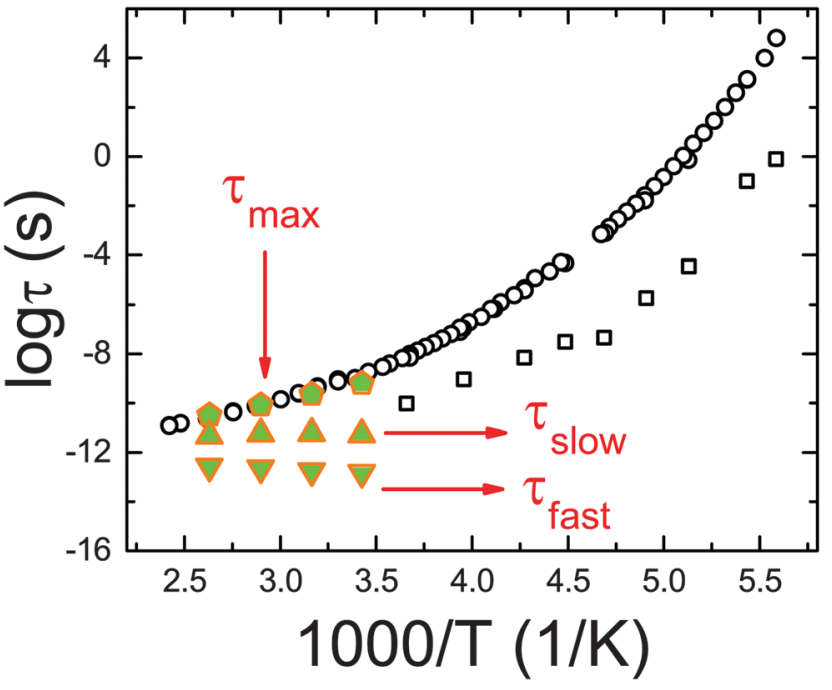

Fig. 6 Relaxation map of glycerol as a function of reciprocal temperature. Open (filled) symbols represent DS (QENS) data. On the scale of this map, relaxation times for the $\mathrm{HT}$ and $\mathrm{HG}$ are indistinguishable.

This, perhaps surprising, insensitivity to temperature has been observed in other prototypical strongly associated liquids like hydrogen fluoride. ${ }^{42,43}$ Whether this behaviour is characteristic of this family of complex liquids remains an open question. On the basis of these considerations, it is unlikely that the $\beta_{\mathrm{JG}}$ relaxation measured by $\mathrm{DS}^{12}$ is related to these dynamical processes probed by QENS (see Fig. 6).

The analysis of the neutron scattering spectra does not only allow us to extract the rates or relaxation times of the processes, but also determine the length scale of the two confined motions. The terms describing these motions of the molecule and tagged as slow and fast, respectively, are given in the scattering function by $\left[P_{i}(Q) \cdot \delta(\omega)+\left(1-P_{i}(Q)\right) L_{P_{i}}\right]$ where $P_{i}=a, b$. In Fig. 7 we present
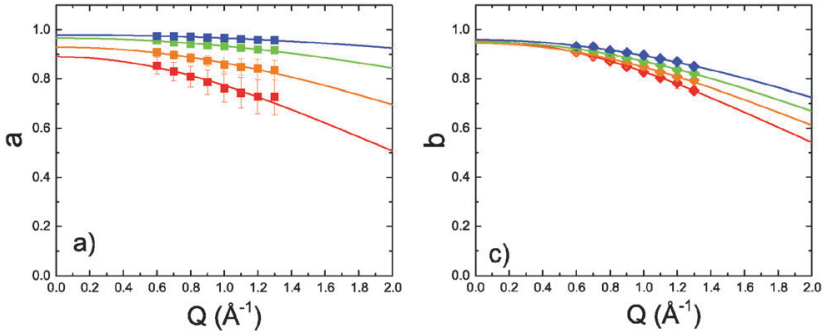

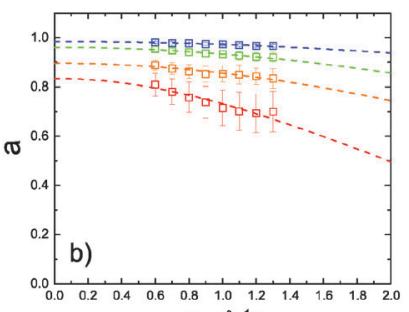

$Q\left(\AA^{-1}\right)$

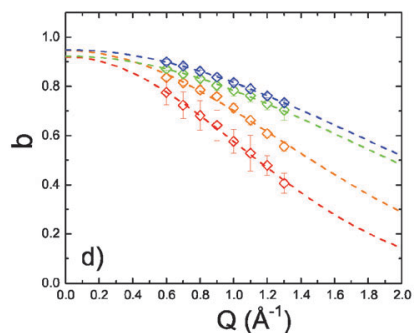

$Q\left(\AA^{-1}\right)$
Fig. 7 Q-dependence of the parameters $a$ (panels $a$ and $b$ ) and $b$ (panels $c$ and d) for each temperature extracted from both the HG model (panels a and $c$ ) and $\mathrm{HT}$ model (panels $\mathrm{b}$ and d). Also shown are fits of $C \exp \left(-\pi^{2} \sigma^{2} Q^{2}\right)$. 

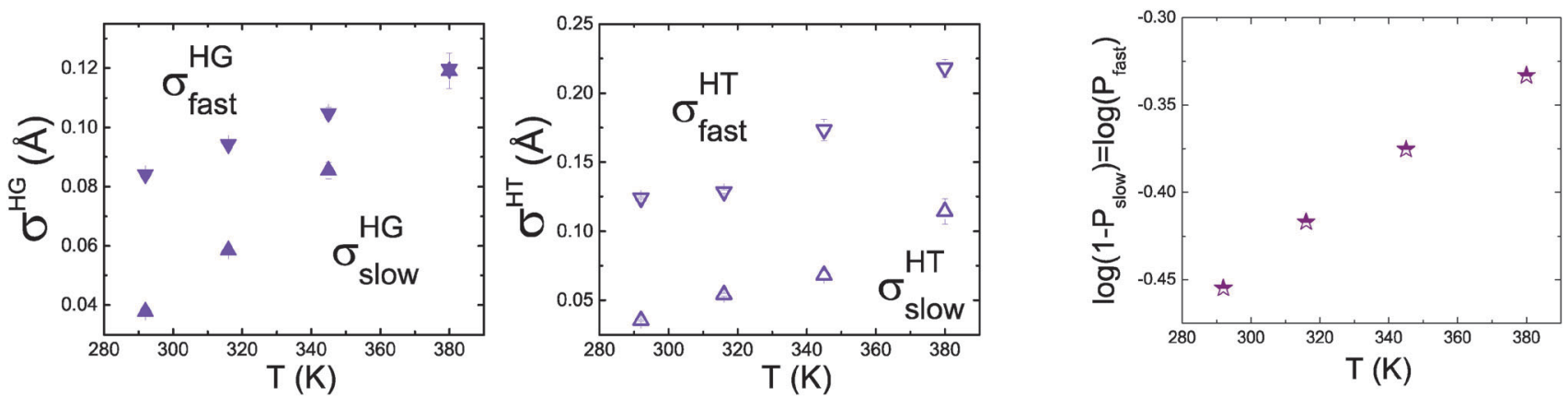

Fig. 8 Two panels on the left: length scales associated with the slow and fast localized relaxation modes of the HG (left) and the HT (middle). The panel on the right shows the proportion of molecules performing the fast motion in the HT scenario.

the $Q$-dependence of parameters $a$ and $b$ for the two models and at all temperatures. These parameters exhibit the same overall behaviour, namely they decrease with increasing $Q$ or $T$. Length scales $\sigma$ associated with slow $\left(\tau_{\text {slow }}\right)$ and fast $\left(\tau_{\text {fast }}\right)$ dynamical processes have been obtained by considering the first term of the cumulant expansion of the structure factor, ${ }^{44,45}$ a Gaussian term in $Q$ that may be written as $C \exp \left(-\pi^{2} \sigma^{2} Q^{2}\right)$. The prefactor $C$ in this cumulant expansion is used to account for multiple-scattering effects, which translate into an effective reduction of the Elastic Incoherent Structure Factor obtained from the QENS data. ${ }^{34}$ The fits to the data can be seen in Fig. 7.

For both the HT and HG, we find that the shorter length scales correspond to "slow" movements taking place in the picosecond time range and longer length scales are associated with "fast" movements. This somewhat counter-intuitive result is physically plausible provided that these two motions conform to different microscopic mechanisms. In this context, the microscopic picture recently proposed by Cicerone et al. ${ }^{4}$ offers a natural explanation of our results (see below). The $\sigma$ values are model dependent both in terms of their magnitude and overall temperature dependence, but in both the HT and HG, they increase with temperature and are invariably larger for faster motions. For the HT, however, both length scales are distinctly different over the entire temperature range investigated in this work, whereas for the HG both length scales converge to the same value at the highest temperature investigated - while the two motions remain well separated with regard to their speed. The HT scenario consists of two populations (slow and fast molecules) the ratio of which shifts with rising temperature in favor of more fast molecules ( $c f$. Fig. 8).

The analysis of the QENS data presented above leads us to the unequivocal conclusion that there are three distinct dynamical processes taking place in liquid glycerol: one associated with the centre-of-mass translational diffusion of the molecule as a whole; and the other two corresponding to localized picosecond (slow) and sub-picosecond (fast) motions, both of which are insensitive to temperature. This result, together with our finding that fast (slow) motions take place over long (short) length scales are independent of the underlying model chosen to fit the experimental data.

Concerning the localized motions, it has to be noted that the information provided by the measured scattering function is not sufficient to establish a clear-cut preference for either physical scenario; the HT is favored over the HG but we cannot exclude that additional information might revert this order. For the less likely HG, all molecules would be subjected to the same two types of motions, which we call rattling and hopping, at all times and also these two mechanisms would need to hold at all temperatures. The (smaller) rattling motion would need to occur on a slower time scale than the (larger) hopping and, at temperatures approaching $T=380 \mathrm{~K}$, the two length scales merge ( $c f$. Fig. 8), although their associated relaxation times are still clearly different (cf. Fig. 6). Our results for the HG scenario at high temperatures are also compatible with the microscopic mechanism proposed by Mamontov, ${ }^{20}$ with rattling motions described by confined diffusion on a sphere and fast motions by intramolecular localized motions. Given the information encoded in our QENS data, the HT is the more plausible option, whereby a sizable and temperature-dependent fraction of the molecular ensemble undergoes faster motions over longer length scales. Following the work of Cicerone et al., ${ }^{4}$ these motions would be associated with molecules belonging to regions in the fluid dominated by collective dynamics. The remainder of the ensemble would then move more slowly over shorter length scales, a contribution that one could tentatively ascribe to "normal-liquid regions". ${ }^{3}$ These motions are well separated in relaxation time over the entire temperature range investigated, and show no signs of merging into a single dynamical process.

\section{Acknowledgements}

This work was supported by the Spanish MINECO (grant no. FIS2011-24439) and by the Government of Catalonia (grant no. 2014SGR-0581). F. F.-A. gratefully acknowledges financial support from the UK Science and Technology Facilities Council. This work is based upon experiments performed at the TOFTOF instrument operated by FRM II at Heinz Maier-Leibnitz Zentrum (MLZ), Garching, Germany.

\section{References}

1 T. B. Schrøder, S. Sastry, J. C. Dyre and S. C. Glotzer, J. Chem. Phys., 2000, 112, 9834.

2 P. Chaudhuri, L. Berthier and W. Kob, Phys. Rev. Lett., 2007, 99, 060604. 
3 H. Tanaka, T. Kawasaki, H. Shintani and K. Watanabe, Nat. Mater., 2010, 9, 324.

4 M. T. Cicerone, Q. Zhong and M. Tyagi, Phys. Rev. Lett., 2014, 113, 117801.

5 Y. Grebemichael, T. B. Schrøder, F. W. Starr and S. C. Glotzer, Phys. Rev. E: Stat., Nonlinear, Soft Matter Phys., 2001, 64, 051503.

6 W. F. Rall and G. M. Fahy, Nature, 1985, 313, 573.

7 D. Li, B. Liu, Y. Liu and C. Chen, Cryobiology, 2008, 56, 114.

8 U. Schneider, R. Brand, P. Lunkenheimer and A. Loidl, Phys. Rev. Lett., 2000, 84, 5560.

9 U. Schneider, R. Brand, P. Lunkenheimer and A. Loidl, J. Non-Cryst. Solids, 1998, 173, 235.

10 P. Lunkenheimer, U. Schneider, R. Brand and A. Loidl, Contemp. Phys., 2000, 41, 15.

11 P. Lunkenheimer, R. Wehn, U. Schneider and A. Loidl, Phys. Rev. Lett., 2005, 95, 055702.

12 P. Lunkenheimer and A. Loidl, Chem. Phys., 2002, 284, 205.

13 A. Brodin and E. A. Rössler, Eur. Phys. J. B, 2005, 44, 3.

14 D. J. Tomlinson, Mol. Phys., 1972, 25, 735.

15 L. J. Burnett and J. F. Harmon, J. Chem. Phys., 1972, 57, 1293.

16 D. Aranghel, V. Tripadus, A. Radulescu, M. Petre, M. O. Dima and C. Petre, J. Optoelectron. Adv. Mater., 2010, 12, 953.

17 K. F. Larsson, Phys. Rev., 1968, 167, 171.

18 C. M. Roland and K. L. Ngai, J. Chem. Phys., 1997, 106, 1187.

19 R. Busselez, R. Lefort, A. Ghoufi, B. Beuneu, B. Frick, F. Affouard and D. Morineau, J. Phys.: Condens. Matter, 2011, 23, 505102.

20 E. Mamontov, Chem. Phys. Lett., 2012, 530, 55.

21 J. Blieck, F. Affouard, P. Bordat, A. Lerbret and M. Descamps, Chem. Phys., 2005, 317, 253.

22 J. Wuttke, I. Chang, O. G. Randl, F. Fujara and W. Petry, Phys. Rev. E: Stat. Phys., Plasmas, Fluids, Relat. Interdiscip. Top., 1996, 54, 5364.

23 J. Wuttke, W. Petry, G. Coddens and F. Fujara, Phys. Rev. E: Stat. Phys., Plasmas, Fluids, Relat. Interdiscip. Top., 1995, 52, 4026.

24 J. Wuttke, J. Hernandez, G. Li, G. Coddens, H. Z. Cummins, F. Pujara, W. Petry and H. Sillescu, Phys. Rev. Lett., 1994, 72, 3052.

25 T. Unruh, J. Neuhaus and W. Petry, Nucl. Instrum. Methods Phys. Res., Sect. A, 2007, 580, 1414.

26 J. Wuttke, FRIDA (fast reliable inelastic data analyis), http:// apps.jcns.fz-juelich.de/doku/frida/start.
27 D. S. Sivia and C. J. Carlile, J. Chem. Phys., 1992, 96, 170.

28 D. S. Sivia and J. Skilling, Data Analysis: A Bayesian Tutorial, Oxford University Press, 2006.

29 L. C. Pardo, M. Rovira-Esteva, S. Busch, J.-F. Moulin and J. L. Tamarit, Phys. Rev. E: Stat., Nonlinear, Soft Matter Phys., 2011, 84, 046711.

30 L. C. Pardo, M. Rovira-Esteva, S. Busch, M. D. Ruiz-Martín and J. L. Tamarit, J. Phys.: Conf. Ser., 2011, 325, 012006.

31 A. Henao, M. Rovira-Esteva, A. Vispa, J. L. Tamarit, E. Guardia and L. C. Pardo, J. Phys.: Condens. Matter, 2013, 25, 454217.

32 T. Kikuchi, K. Nakajima, S. Ohira-Kawamura, Y. Inamura, O. Yamamuro, M. Kofu, Y. Kawakita, K. Suzuya, M. Nakamura and M. Arai, Phys. Rev. E: Stat., Nonlinear, Soft Matter Phys., 2013, 87, 062314.

33 C. P. Lindsey and G. D. Patterson, J. Chem. Phys., 1980, 73, 3348 .

34 S. Busch and T. Unruh, J. Phys.: Condens. Matter, 2011, 23, 254205.

35 L. C. Pardo, M. Rovira-Esteva, S. Busch, J.-F. Moulin and J. L. Tamarit, Phys. Rev. E: Stat., Nonlinear, Soft Matter Phys., 2011, 84, 046711.

36 L. C. Pardo, M. Rovira-Esteva, S. Busch, M. D. Ruiz-Martin and J. L. Tamarit, J. Phys.: Conf. Ser., 2011, 325, 012006.

37 A. Henao, M. Rovira-Esteva, A. Vispa, J. L. Tamarit, E. Guardia and L. C. Pardo, J. Phys.: Condens. Matter, 2013, 25, 454217.

38 G. P. Johari and M. Goldstein, J. Chem. Phys., 1970, 53, 2372.

39 G. P. Johari and N. Y. Ann, Acad. Sci., 1976, 279, 117.

40 T. Unruh, C. Smuda, S. Busch, J. Neuhaus and W. Petry, J. Chem. Phys., 2008, 129, 121106.

41 A. Vispa, M. Rovira-Esteva, M. D. Ruiz-Martín, S. Busch, T. Unruh, L. C. Pardo and J. L. Tamarit, J. Phys.: Conf. Ser., 2014, 549, 012013.

42 F. Fernandez-Alonso, F. J. Bermejo, S. E. McLain, J. F. C. Turner, J. J. Molaison and K. W. Herwig, Phys. Rev. Lett., 2007, 98, 077801.

43 F. Fernandez-Alonso, S. E. McLain, J. W. Taylor, F. J. Bermejo, I. Bustinduy, M. D. Ruiz-Martín and J. F. C. Turner, J. Chem. Phys., 2007, 126, 234509.

44 A. Rahman, K. S. Singwi and A. Sjölander, Phys. Rev., 1962, 126, 986.

45 J. P. Boon and S. Yip, Molecular Hydrodynamics, McGraw-Hill, 1980.

46 These programs can be found at http://gcm.upc.edu/en/ members/luis-carlos/bayesiano. 